\title{
Determining Longitude: A Brief History
}

Jorge Mira-Pérez, and Salvador X. Bará

Citation: Physics Today 58, 10, 15 (2005); doi: 10.1063/1.2138398

View online: https://doi.org/10.1063/1.2138398

View Table of Contents: https://physicstoday.scitation.org/toc/pto/58/10

Published by the American Institute of Physics

\section{ARTICLES YOU MAY BE INTERESTED IN}

Rømer and the Finite Speed of Light

Physics Today 57, 16 (2004); https://doi.org/10.1063/1.1878320

Whence the Force of $\mathrm{F}=\mathrm{ma}$ ? I: Culture Shock

Physics Today 57, 11 (2004); https://doi.org/10.1063/1.1825251

Relativity and the Global Positioning System

Physics Today 55, 41 (2002); https://doi.org/10.1063/1.1485583

Echegaray-Fiscal Scientist and More

Physics Today 53, 76 (2000); https://doi.org/10.1063/1.1306382

The secret of the Soviet hydrogen bomb

Physics Today 70, 40 (2017); https://doi.org/10.1063/PT.3.3524

Domingo de Soto, early dynamics theorist

Physics Today 62, 9 (2009); https://doi.org/10.1063/1.3074244

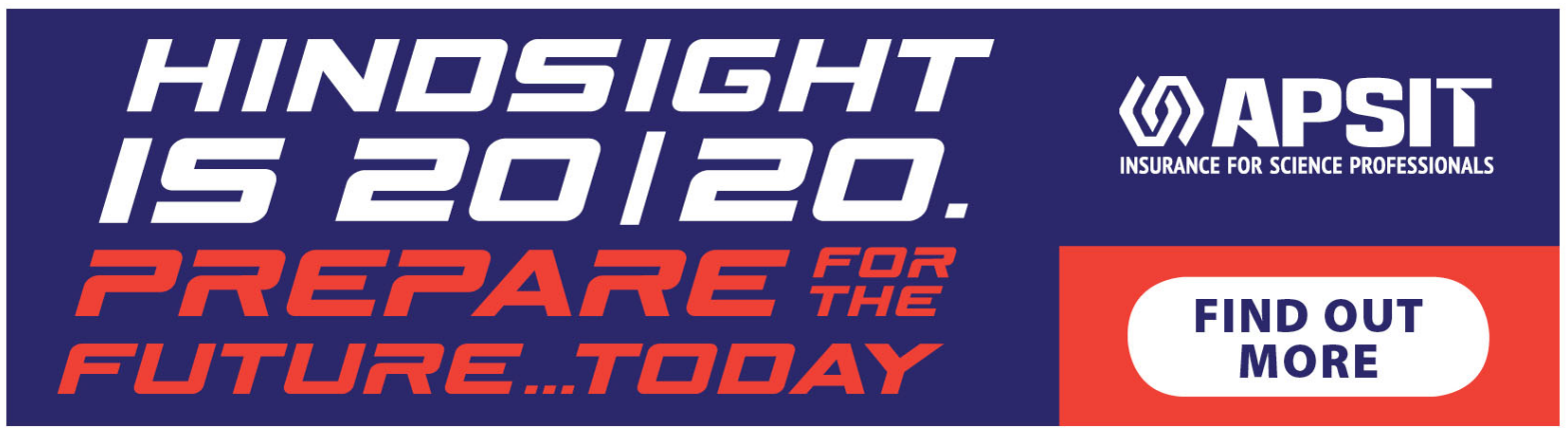


case, however, that this entails conservation of mass. Although the mass of an isolated particle can be determined from its four-momentum, that connection does not apply to multiparticle states. And since particles do interact, and even decay, a result that is only valid for singleparticle states does not allow one to draw general conclusions. That is why the many specific manifestations of nonconservation of mass I discussed in my column can beand are-perfectly consistent with special relativity in general, and with the conservation of fourmomentum in particular.

Frank Wilczek

Massachusetts Institute of Technology Cambridge

\section{Determining Longitude: A Brief History}

$\mathrm{t}$ was a pleasure to read Fokke Tuinstra's letter (PHYSICS TODAY, December 2004, page 16) on Ole Rømer's proof that the speed of light is finite. Perhaps an account of the reasons that led him and other scientists to study so precisely the moons of Jupiter (the system Rømer used for his result) would help com- plete that nice piece of history.

After Christopher Columbus's

1492 trip to America, ships, mostly

Spanish and Portuguese, crossed the Atlantic Ocean in increasing numbers. That migration forced a qualitative change in coastal navigation techniques, since mariners sailed without visible land or fixed points of reference. The determination of geographical longitude became an urgent need-at sea for navigation, and on land for more precise cartography and settlement of territorial disputes.

The need led Spanish King Felipe II (1527-98) to offer a substantial reward to the inventor of a method to "find longitude." The reward amount was increased years later by his successors, and greater rewards were promised by other countries as they began their own oceanic navigations. The British government, for example, offered $£ 20000$ in 1714 to whoever could provide a satisfactory method of finding a ship's position to within half a degree.

What has this circumstance to do with the abrupt and almost obsessive dedication to the study of Jupiter's moons?

When Galileo discovered Jupiter's first four satellites in 1610 , he realized that comparing their eclipse times with local times at a ship's position could be a key component in determining longitude. In 1612, and on three occasions thereafter, Galileo tried unsuccessfully to convince the Spanish monarchy of the usefulness of his method. Although his idea proved to be impractical at sea, it did eventually work on land. For example, the difference in longitude between Paris and Uranienborg, Denmark, was calculated on the basis of the eclipse times taken by Giovanni Cassini, Jean Picard, and Ole Rømer in 1671.

Clockmaker John Harrison won the British prize by developing, between 1735 and 1764, increasingly precise and practical mechanical chronometers, including the one used by James Cook in some of his expeditions.

Rømer's work is probably the first measured Doppler effect; that is, he discovered that the value observed on Earth for the period of Jupiter's moons depends on Earth's velocity relative to Jupiter. It is also noteworthy that today's global positioning system solution to the old problem of finding longitude requires the use of general-relativistic corrections to 


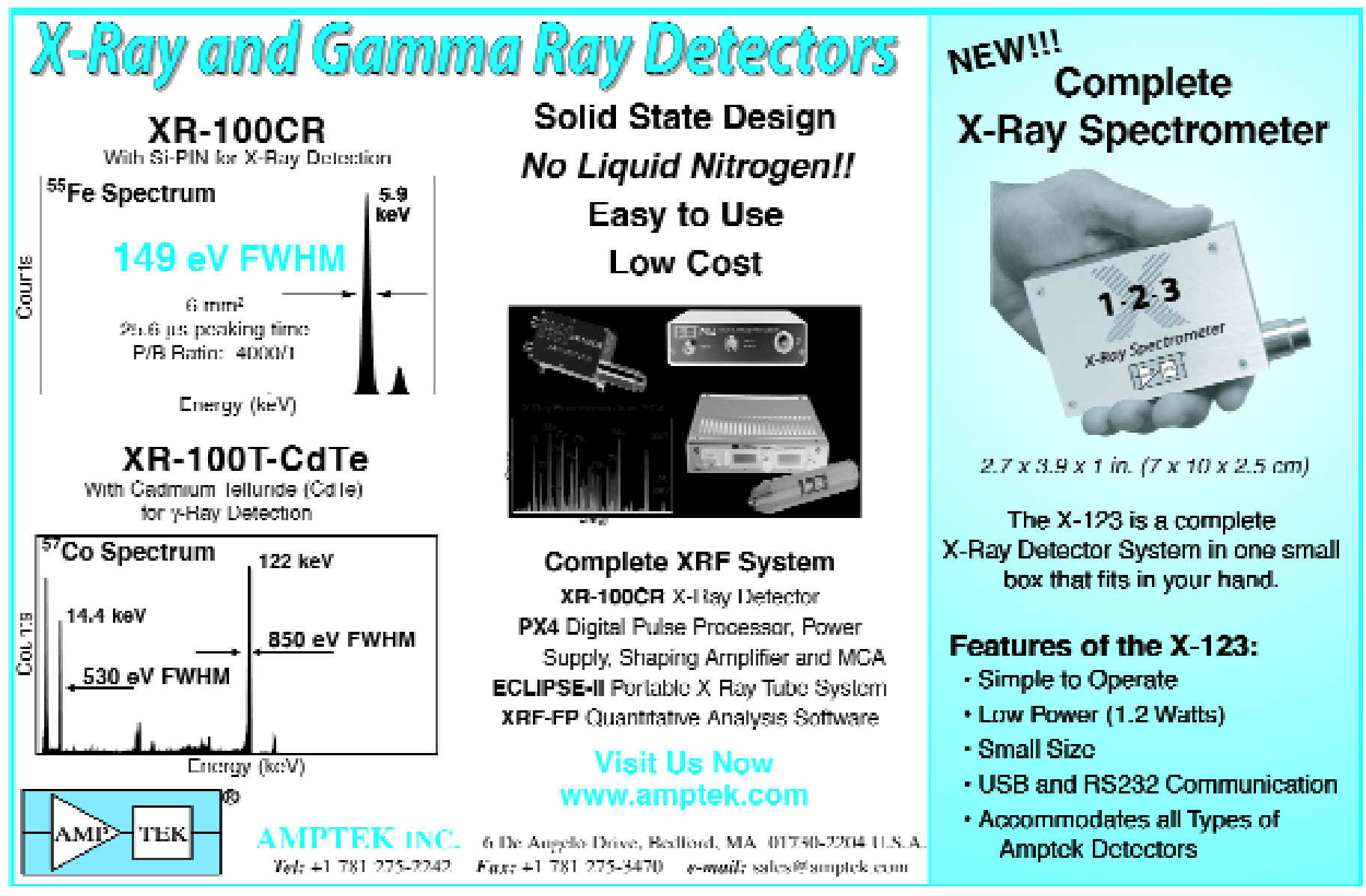

See www.pt.ims.ca/6087-10

attain maximum accuracy. If Rømer's studies are an excellent example of how technologically driven research may provide fundamental basic science results, the GPS application of general relativity also nicely demonstrates complementary feedback in the science-technology system.

Jorge Mira-Pérez (fajmirap@usc.es)

Salvador X. Bará

Universidade de Santiago de Compostela

Santiago de Compostela, Spain

\section{Statistical-Mechanical Viewpoint Needed in Teaching Biophysics \\ o a retired professor of physical} chemistry, the proposals outlined in "Teaching Biological Physics" (PHysics TODAY, March 2005, page 46) concerning the inadequate course content for biophysics majors and possible remedies are rational and merit implementation to various degrees. I followed the proposals of authors Ray Goldstein, Phil Nelson, and Tom Powers up to the paragraph that refers to the "feeling for entropy." I drew back at the totally misleading analogy in their figure 2 .

Molecules, large or small, do not hop over barriers like buffalo in a stampede. Saying, as many instructors do, that a particular chemical conversion occurs because the system is "seeking the lowest chemical potential" and in so doing "it must surmount a free energy barrier" gives students no conceptual content, only words to repeat on an examination.

Furthermore, although the entropy function developed in a course on conventional thermodynamics is fundamental, it resides in a special mental compartment. It remains unconnected to molecular and statistical concepts that provide students with the basis for constructing models of the complex processes they will encounter in biophysical studies. Why not present the molecular and statistical models early in the program?

Second-year physics and biophysics majors have learned the fundamentals of quantum mechanics, and will not reject the assertion that for each species of molecule, small or large, there is a distinctive, huge se- quence of discrete energy states in the form of translations, rotations, vibrations, electronic excitations, and so forth.

Ludwig Boltzmann demonstrated that in any macroscopic sample of matter at equilibrium, the population of accessible states follows a specific temperature-dependent distribution function.

It is useful to consider a feature of the distribution, the density of accessible states, which depends on the total energy and increases rapidly with increasing energy. For the conversion of $A$ to $B$, there must be a sequence of structures that are intermediate between the original and new species. For the intermediates there exist corresponding distribution functions characterized by their distinctive densities of states. Generally, the intermediate structures have higher electronic energies than either A or B, so only the higherenergy states of A or B are close to those of the intermediates and thus have a high probability of making transitions. Clearly, only if B has a higher density of states at that level does the conversion succeed, since the higher density of accessible 